\title{
Idiopathic Phantosmia: Outcome and Clinical Significance
}

\author{
Basile N. Landis Jens Reden Antje Haehner \\ Smell and Taste Clinic, Department of Otorhinolaryngology, University of Dresden Medical School, \\ Dresden, Germany
}

\section{Key Words}

Parosmia · Anosmia · Olfaction • Phantosmia • Parkinson's disease

\begin{abstract}
Background/Aim: Little is known about the clinical significance of phantosmia. The literature on phantosmia indicates that this symptom has a wide range of differential diagnoses. However, most cases of phantosmia remain of unknown origin. Our goal was to follow up patients with idiopathic phantosmia, with special regard to improvement rates and possible severe health conditions preceded by phantosmia of unknown origin. Methods: Forty-four patients with idiopathic phantosmia which had consulted our Ear-Nose-Throat Smell and Taste Clinic over the last 10 years were contacted by telephone and underwent a structured medical interview. Results: None of the patients had developed any severe health condition or Parkinson's disease. More than 5 years after the occurrence of phantosmia, more than $50 \%$ of the patients experienced disappearance (31.8\%) or improvement $(25 \%)$. In the remaining cases, phantosmia did not change $(38.7 \%)$ or became worse $(<5 \%)$. Conclusion: The main findings of the present study were that idiopathic phantosmia improves or disappears in almost two thirds of the patients after more than 5 years, and that idiopathic phantosmia seems to be more likely a harmless symptom rather than a reliable predictor of early Parkinson's disease or other severe diseases.

Copyright $\odot 2010$ S. Karger AG, Basel
\end{abstract}

\section{Introduction}

For clinical purposes, olfactory complaints can be divided into quantitative (e.g. anosmia or hyposmia) and qualitative (e.g. phantosmia or parosmia) disorders, whereas both can occur simultaneously in a given patient. In contrast to hyposmia and anosmia, which are easily assessed by psychophysical and electrophysiological tests, parosmia and phantosmia are only diagnosed based on the patient's history. Currently, no tests are available to investigate the presence of parosmia and phantosmia. Parosmia is the distorted olfactory perception in presence of an odor source, and phantosmia, or olfactory hallucination, is an erroneous odor occurring without any trigger. Parosmia is mostly associated with postinfectious and post traumatic olfactory dysfunction while phantosmia does not seem to be particularly related to any kind of cause of olfactory disorder. In most cases, phantosmia has no detectable origin and is diagnosed as idiopathic phantosmia [1-2].

Several case reports identified idiopathic phantosmia to herald diseases such as tumors, chronic rhinosinusitis, intracranial hemorrhages, epilepsy, psychiatric conditions or, as recently suggested, Parkinson's disease (PD) [3]. In idiopathic hyposmia and anosmia, we could recently show that almost $10 \%$ of patients develop extrapyramidal symptoms or PD 4 years after initial presentation [4]. In light of these recent findings, the goal of the present study was to (1) investigate if any of the patients seen for idiopathic phantosmia at the Smell and Taste Clinic

\section{KARGER}

Fax +4161306 1234 E-Mail karger@karger.ch www.karger.com
Basile N. Landis

Smell and Taste Clinic, Department of Otorhinolaryngology

University of Dresden Medical School, Fetscherstrasse 74

DE-01307 Dresden (Germany)

Tel. +49351 45822 68, Fax +49351 45843 26, E-Mail bnlandis@yahoo.co.uk 
developed $\mathrm{PD}$, other neurodegenerative disorders or any other severe health condition, and (2) to gain further information concerning the spontaneous evolution of the phantosmia symptom.

\section{Methods}

\section{Patients}

The database of the Smell and Taste Clinic of the Otorhinolaryngology Department of the University Medical School of Dresden was searched for all cases of patients which had been diagnosed with 'idiopathic phantosmia' since 2002 . A total of 58 patients could be identified. All patients had been regularly followed up in our Smell and Taste Outpatient Clinic, and details such as patient history and supplementary exams (blood samples and imaging) were reanalyzed in order to assure that no case was likely to be due to any other classical cause of olfactory disorder (e.g. head trauma, upper airway tract infection, or others). The results of the olfactory tests, which were done with the aid of the Sniffin Sticks at initial presentation, were also recorded. This test consists of 3 subtests (threshold, discrimination and identification) forming a composite TDI score, which in the meantime is widely used in Europe for psychophysical testing of olfactory function with recently published and updated norm values [for details, see 5]. According to these TDI values, the patients could be divided into groups suffering from anosmia, hyposmia or showing normosmia besides phantosmia.

\section{Follow-Up}

A trained neurologist and expert in PD (A.H.) contacted all the patients by phone and interviewed them in a structured way, including a detailed patient history, new medications, any kind of health situation changes and especially regarding the presence of extrapyramidal symptoms. Additionally, the patients had to explain whether the phantosmia symptoms had disappeared, improved, remained unchanged or worsened since initial presentation. The study was conducted according to the Declaration of Helsinki on Biomedical Research Involving Human Subjects and approved by the institutional review board.

\section{Statistical Analysis}

Results were analyzed using SPSS 15 for Windows ${ }^{\mathrm{TM}}$ (SPSS Inc., Chicago, Ill., USA). Descriptive statistics were presented as means and standard errors of the mean. One-way ANOVA was used to investigate differences between the mean values of phantosmia duration, age and TDI regarding follow-up evolution (disappearance, improvement, status quo, worsening). Correlation analyses were performed using Spearman analyses. For other nonparametric statistics, $\chi^{2}$ tests were used. The $\alpha$ level was set at 0.05 .

\section{Results}

\section{Patient Characteristics}

Of the 58 identified patients diagnosed with idiopathic phantosmia, 44 patients $(76 \%, 18$ men and 26 women,

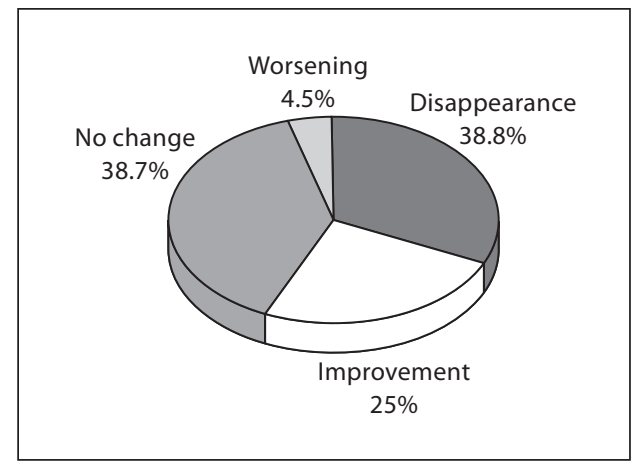

Fig. 1. Percentage of patients who report disappearance, improvement, worsening or no change at all of phantosmia more than 5 years after occurrence.

mean age $59.3 \pm 3.2$ years) could be contacted by phone. The remaining patients either changed address or could not be reached despite several attempts. Male and female patients did not differ regarding mean age (men $60.3 \pm$ 3.5 years, women $58.3 \pm 2.2$ years; $p=0.5$ ), TDI score (men $19.1 \pm 1.5$ points, women $19.6 \pm 1.7$ points; $\mathrm{p}=0.8$ ) or duration of phantosmia (men $6.1 \pm 0.95$ years, women $6.3 \pm 0.7$ years; $p=0.8)$. Fourteen patients had concomitant anosmia (31.8\%), 26 patients had hyposmia (59.1\%) and 4 patients were normosmic (9.1\%). The mean duration of phantosmia was $6.3 \pm 0.9$ years.

\section{Follow-Up}

None of the patients had developed any extrapyramidal symptoms or PD, nor did any other severe health problems (e.g. brain tumor) occur.

Phantosmia symptoms disappeared in 14 patients (31.8\%), improved in 11 patients (25\%), remained the same in 17 patients (38.7\%) and worsened in 2 patients $(4.5 \%$; fig. 1). One-way ANOVA showed that neither TDI score $(\mathrm{F}=1.3, \mathrm{p}=0.2)$, duration of phantosmia $(\mathrm{F}=0.7, \mathrm{p}=0.5)$ nor age $(\mathrm{F}=0.3, \mathrm{p}=0.8)$ were associated with any of the following variables: disappearance, improvement, status quo or worsening. Frequency analysis did not show any factor such as gender ( $\chi^{2}$ test, $\mathrm{F}=1.2$, d.f. $\left.=3 ; \mathrm{p}=0.7\right)$ or TDI score (anosmia, hyposmia, normosmia, $\chi^{2}$ test, $\mathrm{F}=6.9$, d.f. $=6 ; p=0.3)$ to be associated with phantosmia recovery.

\section{Discussion}

The main findings of the present study were (1) that idiopathic phantosmia improves or disappears in more than $50 \%$ of the patients after more than 5 years, and (2) 
that idiopathic phantosmia seems to be more likely a harmless symptom rather than a reliable predictor of early PD or other severe diseases, at least not for the current interval of observation.

Most clinical literature on olfactory disorders is focused on measurable dysfunction such as anosmia and hyposmia [6]; today, reliable data on the frequency, origins and spontaneous recovery rates of quantitative smell disorders exist $[7,8]$. The clinical knowledge of qualitative olfactory disorders is a little different. Systematic investigation of when and how often parosmia occurs revealed that this symptom is mostly linked to postinfectious and posttraumatic olfactory disorders [1] but also occurs in the healthy population, though to a much lesser extent [9]. Parosmia seems to be linked with lower olfactory bulb volumes in patients with hyposmia when compared with hyposmic patients without parosmia [10]. Parosmia progressively and spontaneously disappears over a period of 1.5 years in one third of the patients [11] and only recently has been identified as a positive predictor for spontaneous recovery [12].

The literature on phantosmia is rather sparse with less than 25 articles found in Pubmed when typing this keyword. This is partly due to the fact that many synonyms are used for phantosmia such as olfactory hallucination, unstimulated olfactory distortion or dysosmia. However, extending the search with all these keywords still shows that most articles are case reports with varying etiologies for the described odor phantom. Besides the pioneering work of Leopold and colleagues $[2,13,14]$ who attempted to systematically describe, diagnose and treat phantosmia, little clinically useful knowledge is available to the otorhinolaryngologist. Recently, Reden et al. [1] identified phantosmia to potentially occur in almost all conditions affecting the sense of smell. Amongst investigated cases, idiopathic phantosmia was the most frequent diagnosis.

Very little information is available on how idiopathic phantosmia evolves over time. Deems et al. [15] reported that approximately $10 \%$ of dysosmia patients recover from the distortion. Unfortunately, these authors did neither differentiate between parosmia and phantosmia nor did they specify after what time lapse recovery occurred. Reden et al. [1] reported parosmia to disappear or weaken in $29 \%$ and phantosmia in $53 \%$ of followed-up patients after an observational period of almost 1 year. This is similar to our rate after a mean observation of 6 years, which suggests that most phantom smells disappear within the first year, and very little supplementary recovery can be expected after this period.
Since curative treatment for olfactory disorders is not always possible, serious counseling concerning possible prognosis and evolution becomes more important [16]. With the present data we hope to provide valuable prognostic information when it comes to give possible outlooks for symptom evolution to patients with phantosmia. In light of the fact that qualitative olfactory disorders such as parosmia and phantosmia may affect the quality of life [17], it appears to be helpful for physicians to tell the patient that in more than half of the cases phantosmia will disappear completely or improve and that phantosmia is not a common predictor of PD as it seems to be the case for unexplained hyposmia [18]. Compared with parosmia, it has to be noted that the time lapse between occurrence and improvement seems to be similar, with phantosmia taking almost a year and parosmia 14 months in average to improve $[1,11]$.

It has to be kept in mind that the present study did not identify gender, age or measurable olfactory function at initial presentation to be considered as positive or negative predictive factors for improvement of phantosmia. Vice versa, parosmia but not phantosmia has recently been shown to be a positive predictor of olfactory recovery [12].

In qualitative gustatory symptoms, generally subsumed as dysgeusia, it has been shown that the depressive state is associated with low recovery rates [19]. Based on the findings of Deems et al. [19] in dysgeusia patients, it would be interesting to investigate whether this is also true for phantosmia in general.

The second aim of the present study was to follow up patients with phantosmia of unknown origin in order to check whether this symptom is an early sign of PD or possibly other diseases. Recent reports have shown that some patients who developed PD have experienced phantosmia very early in the course of the disease [3, 20,21]. However, phantosmia seems to be rather rare in PD. In contrast, anosmia and hyposmia are present in more than $90 \%$ of PD patients in very early disease stages [22, 23]. Moreover, almost $10 \%$ of patients with olfactory loss or a decrease of unknown origin have been shown to develop PD or suspected PD within the first 4 years after initial presentation [4]. In contrast to what could have been expected in light of these recent findings, none of the patients with idiopathic phantosmia developed PD or any other serious health conditions. Idiopathic phantosmia as an early sign of PD seems to remain a rather exceptional presentation whereas the great majority of people with idiopathic phantosmia will not develop PD.

However, the present study has the shortcoming of being a telephone survey rather than a physical re-exami- 
nation, and the investigated sample is rather small. This is largely due to the fact that our Smell and Taste Outpatient Clinic included a nationwide recruitment with most patients living far away from the investigating Smell and Taste Clinic. Consequently, the present data are appeasing when it comes to possible health conditions associated with idiopathic phantosmia. Nevertheless, we do not emphasize for general relaxation when it comes to the routine workup of idiopathic phantosmia, which remains a symptom to be taken seriously and where brain magnetic resonance imaging must exclude the presence of any brain tumor.

\section{Conclusion}

Idiopathic phantosmia seems to have an improvement rate of more than $50 \%$ over a 5 -year period. Further, based on the present data, there is no evidence for idiopathic phantosmia to be a frequent early symptom of any severe health condition. Information on the nature of the disorder and its prognosis is essential in counseling patients suffering from phantosmia.

\section{Acknowledgement}

The study was supported by a grant of the Swiss National Fund for Scientific Research (SSMBS grant No. PASMA-119579/1) to B.N.L.

\section{References}

1 Reden J, Maroldt H, Fritz A, Zahnert T, Hummel T: A study on the prognostic significance of qualitative olfactory dysfunction. Eur Arch Otorhinolaryngol 2007;264: 139-144.

2 Leopold D: Distortion of olfactory perception: diagnosis and treatment. Chem Senses 2002:27:611-615.

3 Landis BN, Burkhard PR: Phantosmias and Parkinson disease. Arch Neurol 2008;65: 1237-1239.

4 Haehner A, Hummel T, Hummel C, Sommer U, Junghanns S, Reichmann H: Olfactory loss may be a first sign of idiopathic Parkinson's disease. Mov Disord 2007;22:839-842.

5 Hummel T, Kobal G, Gudziol H, MackaySim A: Normative data for the 'Sniffin' Sticks' including tests of odor identification, odor discrimination, and olfactory thresholds: an upgrade based on a group of more than 3,000 subjects. Eur Arch Otorhinolaryngol 2007;264:237-243.

6 Doty RL, Bartoshuk LM, Snow JBJ: Causes of olfactory and gustatory disorders; in Getchell TV, Doty RL, Bartoshuk LM, Snow JBJ (eds): Smell and Taste in Health and Disease. New York, Raven Press, 1991, pp 449-462.

7 London B, Nabet B, Fisher AR, White B, Sammel MD, Doty RL: Predictors of prognosis in patients with olfactory disturbance. Ann Neurol 2008;63:159-166.

8 Reden J, Mueller A, Mueller C, Konstantinidis I, Frasnelli J, Landis BN, Hummel T: Recovery of olfactory function following closed head injury or infections of the upper respi- ratory tract. Arch Otolaryngol Head Neck Surg 2006; 132:265-269.

9 Nordin S, Bramerson A, Millqvist E, Bende M: Prevalence of parosmia: the Skövde population-based studies. Rhinology 2007;45: $50-53$.

10 Mueller A, Rodewald A, Reden J, Gerber J, von Kummer R, Hummel T: Reduced olfactory bulb volume in post-traumatic and postinfectious olfactory dysfunction. Neuroreport $2005 ; 16: 475-478$

11 Faulcon P, Portier F, Biacabe B, Bonfils P. Anosmie secondaire à une rhinite aiguë: sémiologie et évolution à propos d'une série de 118 patients. Ann Otolaryngol Chir Cervicofac 1999;116:351-357.

12 Hummel T, Lotsch J: Prognostic factors of olfactory dysfunction. Arch Otolaryngol Head Neck Surg 2010;136:347-351.

13 Leopold DA, Schwob JE, Youngentob SL, Hornung DE, Wright HN, Mozell MM: Successful treatment of phantosmia with preservation of olfaction. Arch Otolaryngol Head Neck Surg 1991;117:1402-1406.

14 Leopold DA, Loehrl TA, Schwob JE: Longterm follow-up of surgically treated phantosmia. Arch Otolaryngol Head Neck Surg 2002;128:642-647.

15 Deems DA, Doty RL, Settle RG, Moore-Gillon V, Shaman P, Mester AF, Kimmelman CP, Brightman VI, Snow JB Jr: Smell and taste disorders, a study of 750 patients from the University of Pennsylvania Smell and Taste Center. Arch Otolaryngol Head Neck Surg 1991;117:519-528.
16 Landis BN, Stow NW, Lacroix JS, Hugentobler M, Hummel T: Olfactory disorders: the patients' view. Rhinology 2009;47:454459.

17 Frasnelli J, Hummel T: Olfactory dysfunction and daily life. Eur Arch Otorhinolaryngol 2005;262:231-235.

18 Berendse HW, Booij J, Francot CM, Bergmans PL, Hijman R, Stoof JC, Wolters EC: Subclinical dopaminergic dysfunction in asymptomatic Parkinson's disease patients' relatives with a decreased sense of smell. Ann Neurol 2001;50:34-41.

19 Deems DA, Yen DM, Kreshak A, Doty RL: Spontaneous resolution of dysgeusia. Arch Otolaryngol Head Neck Surg 1996;122:961963.

20 Hirsch AR: Parkinsonism: the hyposmia and phantosmia connection. Arch Neurol 2009; 66:538-539; author reply 539.

21 Singh S, Schwankhaus J: Olfactory disturbance in Parkinson disease. Arch Neurol 2009;66:805; author reply 805-806.

22 Haehner A, Boesveldt S, Berendse HW, Mackay-Sim A, Fleischmann J, Silburn PA, Johnston AN, Mellick GD, Herting B, Reichmann H, Hummel T: Prevalence of smell loss in Parkinson's disease - a multicenter study. Parkinsonism Relat Disord 2009;15:490494

23 Ponsen MM, Stoffers D, Twisk JW, Wolters E, Berendse HW: Hyposmia and executive dysfunction as predictors of future Parkinson's disease: a prospective study. Mov Disord 2009;24:1060-1065. 